\title{
Qui guérit a raison?
}

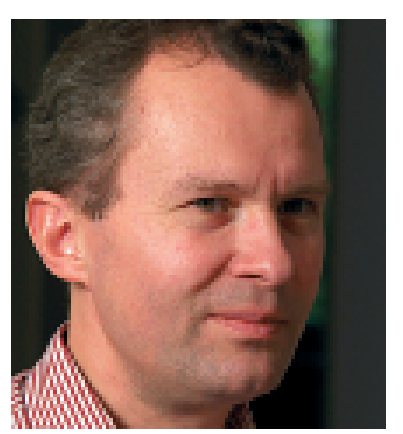

* Prof. Christoph Rehmann-Sutter, Dr ès lettres, biologiste diplômé, directeur du Service d'éthique dans les sciences biologiques de l'Université de Bâle, président de la Commission nationale d'éthique et membre du comité de rédaction du BMS pour les questions d'éthique
Un cas a fait grand bruit dans la presse suisse: celui des enfants genevois Elodie et Noah. Le petit Noah souffrait de granulomatose, une maladie héréditaire du système immunitaire. Il a pu être traité avec succès grâce à une transplantation de moelle épinière de sa sœur Elodie, âgée d'un an. La petite fille a cependant été conçue spécialement à cet effet - c'est là le hic - grâce au recours au diagnostic préimplantatoire (DIP), interdit en Suisse. Les parents ont dû se rendre à Bruxelles pour un traitement de fécondation in vitro. En relatant l'affaire d'Elodie et Noah, la presse a donné un aspect concret au DIP, jusqu'ici abstrait. On peut ainsi s'identifier à la douleur du garçon et aux soucis des parents tout en pouvant accepter pourquoi un tel plan doit être moralement concevable pour les personnes touchées.

Deux aspects ressortent de cette perspective nouvelle née d'une identification. Le premier est la responsabilité morale des parents envers l'enfant malade. Si la seule chance d'aider un enfant gravement atteint est de concevoir un nouvel enfant par le DIP et transplanter au premier des cellules saines, il n'est guère possible aux parents de dire non. Leurs scrupules moraux contre la sélection d'embryons devraient être si hauts qu'ils dépasseraient la valeur de la vie de leur enfant. C'est bien sûr impensable. Le second aspect est qu'il est naturellement évident que le bébé «sauveur» sera aimé pour soi et que la famille accepte Elodie comme un membre à part entière de la famille. Le problème de $l^{\prime}$ «instrumentalisation» est si éloigné des préoccupations concrètes des parents qu'il est hors de question qu'ils le perçoivent, hors qu'il est des réalités telles qu'ils les vivent. Leur monde est marqué de réelle sollicitude et de responsabilité, à l'égard de leur dernière-née également. On ne saurait parler ici $\mathrm{d}^{\prime}$ «utiliser et de jeter».

J'ai assumé plusieurs fois le rôle d'expliquer aux médias que, hormis la perspective interne à une situation, il en existait une autre dite de société, et que, partant d'un cas isolé heureux, on ne saurait promulguer une règle. J'ai trouvé la situation particulièrement difficile, parce que la limite entre abstrait et concret se révèle aussi être une limite de la perceptibilité. Que peut-on se représenter concrètement, si l'on songe à ces implications de «société»? Même si l'on prétend s'y essayer sérieusement, rien n'est aussi simple et lumineux que le cas des enfants Elodie et Noah. Pour moi, les doutes qui planent sur le côté sociétal sont deux: le manque de limites et la pression morale. A mon avis, le DPI est en soi défendable sur le plan éthique s'il s'agit d'empêcher une grave maladie de toucher un enfant à naître. Je considère en effet le diagnostic embryonnaire avant la nidation moins difficile à comprendre et lourd à porter qu'une grossesse imposée à l'essai avec amniocentèse et avortement éventuel à la clé.

Si l'on accepte que le DPI soit pratiqué au bénéfice de tiers, il sera difficile de le limiter à des cas comme ceux d'Elodie et Noah. En effet, pourquoi l'enfant nouvellement conçu devrait-il n'aider qu'un frère ou une sœur? Pourquoi pas la mère, le père ou une amie proche? Pourquoi l'aide se limiterait-elle à un don de moelle? Pourquoi n'incluerait-elle pas une partie du foie? Ou un rein? Quelle étendue d'hospitalisation et quel degré de renoncement devrait-on imposer à cette nouvelle vie? Peut-on, tant qu'à faire, tolérer des formes plus étendues d'ingérence? Afin de cerner ces questions, on ne doit pas penser en termes abstraits, mais se représenter des cas similaires dont les éléments se distinguent plus ou moins les uns les autres. Le deuxième doute est la pression morale qui s'exerce sur les parents. C'est une objection qui se comprend aisément si l'on songe à quel point il est difficile, sinon impossible pour les parents de trouver des arguments convaincants pour refuser l'offre qui leur est faite si elle existe. Voulons-nous une société dans laquelle on se sentirait contraint de concevoir un nouvel enfant pour des raisons thérapeutiques?

Sur ce point, la médiatisation de ce cas concret, qui l'a rendu visible, est des plus instructives. Leçon à en tirer: il permet de poursuivre et élargir la réflexion en restant dans le concret.

Christoph Rehmann-Sutter * 